\title{
Surgical treatment for pelvic giant cell tumor: a multi-center study
}

\author{
Kai Zheng ${ }^{1,2,7}$, Xiuchun $\mathrm{Yu}^{1,2,7^{*}}$, Yongcheng $\mathrm{Hu}^{3,7^{*}}$, Zhen Wang ${ }^{4,7}$, Sujia $\mathrm{Wu}^{5,7}$ and Zhaoming $\mathrm{Ye}^{6,7}$
}

\begin{abstract}
Background: The purpose of this study was to discuss the clinical results which related to the location of giant cell tumors (GCTs) in the pelvis so as to determine the ideal surgical treatment protocol.

Methods: We report 29 cases who accepted surgical treatment from five clinical centers during the last 12 years. All patients were divided into three groups according to tumor locations, and they were also classified into two groups in light of surgical treatments. The parameters for outcome evaluation consisting of general condition, surgical complications, local disease control, and Musculoskeletal Tumor Society (MSTS) 93 functional score had been analyzed, respectively.

Results: Surgical treatment in the acetabular area usually resulted in postoperative complications and poor function. One patient who accepted intralesional surgery and two who accepted wide resection had local recurrence. The mean functional score was 25.4 for the 8 patients who received intralesional surgery and 21.9 for the 21 patients who received wide resection. Surgical complications occurred in 1 patient who underwent intralesional surgery and the other 6 patients who underwent wide resection.

Conclusions: We conclude that surgical treatment of pelvic GCTs in the acetabular area is difficult to select as it is always accompanied by complications and poor function. Compared to wide resection, intralesional surgery combined with a meticulous preoperative planning may lower the recurrence rate and obtain favorable postoperative functional results.
\end{abstract}

Keywords: Giant cell tumor, Pelvis, Surgical treatment

\section{Background}

Giant cell tumors (GCTs) of the bone represent 3-8 \% of all primary bone tumors [1-3], most of which occur at the ends of long bones. Accounting for only 1.5 to $6.1 \%$ of bone GCTs [4-6], pelvic GCTs are rare lesions and no more than 100 cases have been reported in the past five decades before 2004 [7]. In the limited number of literature, Guo et al. reviewed 27 cases with pelvic GCTs, which is the largest series that had ever been reported [8]. To our best knowledge, a multi-center clinical study of GCTs has not been available in any publications. In this study, we collected the data of 29 cases from five clinical institutions to analyze the correlation between the location of GCTs in the pelvis and the

\footnotetext{
* Correspondence: yxch48@vip.sina.com; yongchenghu@126.com

${ }^{1}$ Second Military Medical University, Shanghai, China

${ }^{3}$ Department of Bone Oncology, Tianjin Hospital, Tianjin, China

Full list of author information is available at the end of the article
}

clinical results and to discuss the selection of surgical treatment options.

There is still no widely held consensus in respect to the ideal surgical treatment protocol for giant cell tumors of the pelvis owing to their infrequent occurrence. Due to the complex anatomy of the pelvis and mostly delayed presence in clinical institution, the treatment for pelvic GCTs might be challenging without a standard treatment protocol for consultation. Treatment options for pelvic GCTs include denosumab [9], serial embolization [10], interferon [11], radiation therapy [12], and intralesional curettage or wide resection $[7,13-16]$. Although some primary treatments are favorable for GCT control, it is still necessary to perform tumor resection if the lesion has become resectable. Strengths of intralesional curettage include preservation of pelvic integrity and avoidance of nerve disturbance, but it is also deemed to increase the risk of local recurrence, 
especially for aggressive benign tumors [7, 13-16]. Although wide resection is reported to minimize the chance of local recurrence, this procedure seems to have certain disadvantages such as long-time operation, increased risk of nerve injury and infection, and prosthesis-related complications from hip reconstruction [17-19]. Some suggestions have been given in literatures by retrospective analysis of their own treated patients, but it is hard to say the treatments are not influenced by their preference. Given the multi-center study of pelvic GCTs has not been reported in any literatures, in this study, we reviewed 29 cases treated by five bone tumor experts from five institutions, which hopefully provides information of great value to orthopedic surgeons and assist them to select the optimal treatment protocol from various options.

\section{Methods}

Five hundred thirteen patients with histologically benign GCT of the bone were treated at these five institutions from 2001 to 2013, of which 29 patients who presented with pelvic GCT were retrospectively reviewed. In this study, we included patients with the following criteria: (1) pathological diagnosis of GCT was definite; (2) GCT involved the pelvis but not the sacrum; (3) no prior treatments of the tumor; and (4) complete clinical, radiographic, and pathologic records. Most patients were aged between the third and fourth decades of life at first diagnosis, with a mean age of 39.2 years (range 1557 years). In this series, there were 14 males and $15 \mathrm{fe}-$ males with a mean follow-up period of 59 months (range 18-156 months) (Table 1). Eight patients were treated with intralesional surgery while 21 underwent wide resection. The patients' data was collected from patient records, surgical protocols, and histological and radiological findings. The last follow-up was done via followup exams after surgery or telephone contact.

The research was carried out according to the principles set out in the Declaration of Helsinki 1964 and all subsequent revisions. Informed consent was obtained, and the relevant institutional review board (Jinan Military General Hospital Ethics Committee) had approved the study.

The diagnosis of GCT was established based on the clinical data and imaging studies and confirmed by needle biopsy or open biopsy before surgery as well as pathology examination after surgery. In accordance with the classification system for pelvic tumors by Enneking and Dunham [17], further modified by Sanjay et al. [16], the locations of tumor were divided into three types: type I (ilium), type II (acetabulum), and type III (pubis/ischium). This study included type I lesions in 10 patients, type II in 11 patients, and type III in 8 patients. Radiographical classification system of Campanacci [20] categorizes the lesion into three grades in which grade I indicates an intraosseous lesion, grade II denotes an intraosseous lesion with cortical thinning and expansile borders, and grade III refers to a lesion extending extraosseously and forming a soft tissue mass. There were 5 patients with grade I lesions, 15 patients with grade II lesions, and 9 patients with grade III lesions included in this series. The clinical results were statistically analyzed in light of different GCT locations in the pelvis.

The treatment regimens were classified into group A and group B, and group A had been further divided into two subgroups. Group A1 included 5 patients treated by intralesional curettage, of which two cases applied bone cement to fill defect after curettage. Group A2 included 3 patients treated by microwave inactivation for the GCT lesion before intralesional curettage, after which defects were filled with autograft and allogeneic bone graft (Fig. 1). Twenty-one patients treated by wide resection were included by group B, of which 3 patients involving region I underwent reconstruction of the pelvic ring after resection (Fig. 2) and 5 patients involving region II underwent rod fixation and total hip arthroplasty (Fig. 3). One patient in group B involving region II accepted bone cement filling because of small bone defect, while another patient involving region II accepted femoral head exclusion after resection of pelvic tumors around the acetabulum. The other 11 patients in group $B$ including 5 patients in region $I$ and 6 patients in region III did not receive any reconstruction after resection. The embolization procedure was not performed in some cases as the tumor side of the common iliac artery was temporarily blocked by a vascular clamp to control bleeding during surgery. The effectiveness has been documented that the common iliac artery was mobilized and encircled with nylon tape for temporary occlusion during removal of the tumor [21]. Patients who accepted microwave inactivation before curettage did not have blood transfusion while most of the others had. Adjuvant therapy such as chemotherapy or radiotherapy was not used in the initial treatment in this series. One patient was treated with radiotherapy for the recurrent tumor after surgery.

All patients had been requested to reexamine every month for half a year after surgery, every 3 months in $0.5-2$ years after surgery, and annually after 2 years. The complications and local disease control were recorded each time. Local recurrence was suspected initially by evidence of new bone involvement assessed by radiographs or computerized tomography (CT), and a biopsy was further performed to confirm the suspicion. Musculoskeletal Tumor Society (MSTS) 93 score [22] had been used for functional evaluation at the last follow-up in our study. The MSTS 93 score measures patient activity, including pain, function, emotional acceptance, supports, walking ability, and gait. Each of these six variables was 
Table 1 Clinical data and surgical results for 29 patients with GCTs involving the pelvis

\begin{tabular}{|c|c|c|c|c|c|c|c|c|c|}
\hline No. & $\begin{array}{l}\text { Gender/ } \\
\text { age (years) }\end{array}$ & Location $^{\mathrm{a}}$ & Grade $^{b}$ & Treatment & Reconstruction & Complication & $\begin{array}{l}\text { Follow-up } \\
\text { (months) }\end{array}$ & $\begin{array}{l}\text { Function } \\
\text { (MSTS 93) }\end{array}$ & $\begin{array}{l}\text { Recurrence or } \\
\text { metastasis }\end{array}$ \\
\hline 1 & $F / 39$ & $1+\|$ & III & Wide resection & Rod fixation + THA & & 24 & 19 & Recurrence \\
\hline 2 & $M / 34$ & $1+\|$ & III & Wide resection & Rod fixation + THA & $\begin{array}{l}\text { Wound healing } \\
\text { disturbance }\end{array}$ & 136 & 22 & No \\
\hline 3 & $F / 33$ & । & $\|$ & Wide resection & No & & 63 & 26 & No \\
\hline 4 & $F / 42$ & $1+\|$ & III & Wide resection & Rod fixation + THA & $\begin{array}{l}\text { Wound healing } \\
\text { disturbance }\end{array}$ & 12 & 17 & Recurrence \\
\hline 5 & $M / 47$ & III & । & Wide resection & No & & 36 & 27 & No \\
\hline 6 & $M / 42$ & $\|+\| I$ & III & Wide resection & Rod fixation + THA & & 22 & 16 & No \\
\hline 7 & $F / 43$ & $\|$ & $\|$ & Intralesional curettage & No & Limb shortening & 156 & 18 & No \\
\hline 8 & $F / 25$ & । & $\|$ & Wide resection & No & & 140 & 25 & No \\
\hline 9 & $M / 37$ & $1+\|$ & III & Intralesional curettage & Cement & & 6 & 23 & Recurrence \\
\hline 10 & $M / 54$ & III & $\|$ & Wide resection & No & & 56 & 22 & No \\
\hline 11 & $M / 57$ & III & I & Wide resection & No & & 53 & 23 & No \\
\hline 12 & $M / 41$ & $\|+\|$ & III & Wide resection & Cement & Delayed infection & 49 & 18 & No \\
\hline 13 & $F / 41$ & $\|$ & III & Wide resection & No & Dislocation & 38 & 14 & No \\
\hline 14 & $F / 15$ & $\|+\| I$ & III & Microwave + curettage & Bone graft & & 148 & 28 & No \\
\hline 15 & $F / 34$ & $\|$ & III & Microwave + curettage & Bone graft & & 37 & 26 & No \\
\hline 16 & $F / 42$ & III & III & Microwave + curettage & Bone graft & & 21 & 28 & No \\
\hline 17 & $\mathrm{M} / 50$ & III & $\|$ & Wide resection & No & & 18 & 26 & No \\
\hline 18 & $M / 27$ & III & III & Wide resection & No & & 30 & 24 & No \\
\hline 19 & $M / 46$ & III & $\|$ & Wide resection & No & & 37 & 26 & No \\
\hline 20 & $M / 37$ & $\|+\|$ & III & Wide resection & Rod fixation + THA & $\begin{array}{l}\text { Wound healing } \\
\text { disturbance }\end{array}$ & 18 & 21 & No \\
\hline 21 & $\mathrm{~F} / 50$ & । & $\|$ & Wide resection & $\begin{array}{l}\text { Pelvic ring } \\
\text { reconstruction }\end{array}$ & & 22 & 24 & No \\
\hline 22 & $F / 41$ & । & $\|$ & Wide resection & No & & 26 & 22 & No \\
\hline 23 & $M / 33$ & । & III & Wide resection & $\begin{array}{l}\text { Pelvic ring } \\
\text { reconstruction }\end{array}$ & & 28 & 20 & No \\
\hline 24 & $F / 30$ & । & $\|$ & $\begin{array}{l}\text { Intralesional } \\
\text { curettage }\end{array}$ & Cement & & 93 & 24 & No \\
\hline 25 & $F / 39$ & I & $\|$ & Intralesional curettage & No & & 85 & 28 & No \\
\hline 26 & $M / 32$ & । & III & Wide resection & $\begin{array}{l}\text { Pelvic ring } \\
\text { reconstruction }\end{array}$ & $\begin{array}{l}\text { Wound healing } \\
\text { disturbance }\end{array}$ & 77 & 21 & No \\
\hline 27 & $M / 34$ & । & III & Wide resection & No & & 60 & 22 & No \\
\hline 28 & $F / 38$ & III & $\|$ & Intralesional curettage & No & & 56 & 28 & No \\
\hline 29 & $\mathrm{~F} / 55$ & । & $\|$ & Wide resection & No & & 28 & 24 & No \\
\hline
\end{tabular}

$M$ male, $F$ female, THA total hip arthroplasty

${ }^{\mathrm{a}}$ According to the classification system of Enneking and Dunham

${ }^{\mathrm{b}}$ According to the Campanacci grading system

assessed on a five-point scale, giving a maximum score of 30 points. To some extent, higher MSTS score signifies better functional results.

\section{Results}

Group A1: intralesional curettage (5 cases)

Group A1 was the simplest treatment regimen that intralesional curettage was followed by bone cement filling of the defect (cases 9 and 24) or not (cases 7, 25, and 28). Three patients (cases 24, 25, and 28) demonstrated good functions and were disease-free at followups. One patient (case 9) with ilium and acetabulum lesion who was primarily treated by intralesional curettage and cementation suffered local recurrence 6 months after surgery. The recurrence was confirmed by biopsy, and he refused additional treatments although there is a possibility of reoperation. He died of unclear cause without any imaging and pathology 2 months later. One 

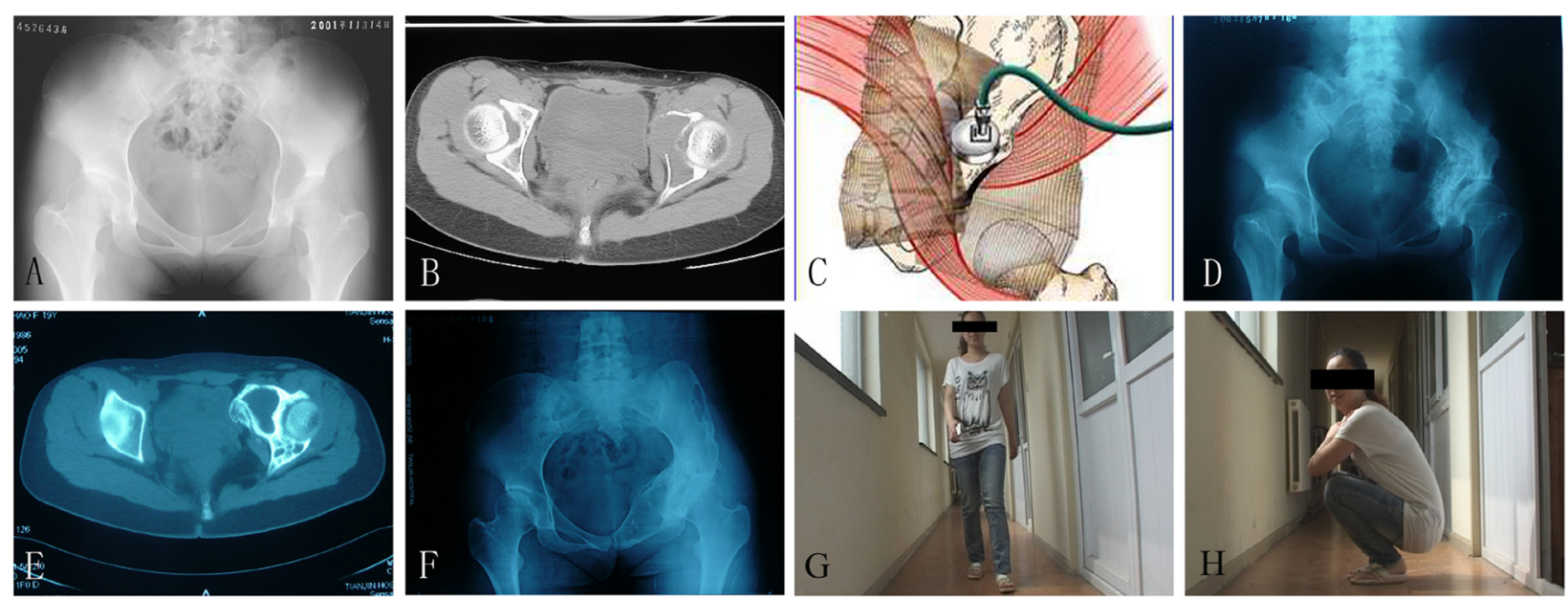

Fig. 1 a Plain film of a 15-year-old female demonstrates an osteolytic lesion in the left periacetabular region. $\mathbf{b} \subset$ $T$ scan presents the tumor involvement in the acetabulum, but the acetabulum cartilage and bone below it can be preserved. c Sketch illustrates that microwave inactivation was employed for this patient before intralesional curettage. $\mathbf{d}$ Plain film at 6 months after operation shows a suspicious lesion in the left pubis. No treatment was performed as there is no presentation of pain or other discomfort. $\mathbf{e} \subset T$ scan at 4 years after operation manifests that the intumescent lesion in the left pubis has mineralized edge. $\mathbf{f}$ Plain film at 11 years after operation shows no recurrence. $\mathbf{g}, \mathbf{h}$ The patient had good function without any discomfort in the last follow-up

patient (case 7) with acetabulum lesion underwent limb shortening. At 3-year follow-up, the patient was free from pain, but she limped and leaned on a cane for longer walks due to the 5-cm shortening. As the shortening gradually worsened, total hip arthroplasty was undertaken for her at 5 years after primary surgery.

\section{Group A2: microwave inactivation for the GCT lesion before intralesional curettage ( 3 cases)}

Three patients (cases 14, 15, and 16) with partial acetabulum lesion underwent microwave inactivation before intralesional curettage, after which defects were filled with autograft and allogeneic bone graft. They remained diseasefree and had no functional limitation at follow-ups. No recurrence and complications were found in this group.

\section{Group B: wide resection (21 cases)}

The 5 patients with tumors of region I (cases 3, 8, 22, 27 , and 29) were treated by wide resection of the iliac tumor without reconstruction whereas the other 3 patients with tumors of region I (cases 21, 23, and 26) were treated by wide resection with pelvic ring reconstruction. The 6 patients with tumors of region III (cases 5, 10, 11, 17,18 , and 29) underwent wide resection without reconstruction. The 5 patients with tumors of region II (cases $1,2,4,6$, and 20) received wide resection with rod fixation and total hip arthroplasty whereas the other 2 patients (cases 12 and 13) received wide resection without reconstruction. One patient (case 4) who had wide resection suffered local recurrence at 12 months after surgery, and she accepted hemipelvectomy after biopsy confirmation as well as radiation therapy for pulmonary metastasis 2 months after hemipelvectomy. Unfortunately, she died of metastasis. One patient (case 1) who underwent resection of the tumor in regions I and II recurred in region III at 18 months after surgery, and she died of unclear cause at half a year after recurrence without additional treatments and imaging examination.
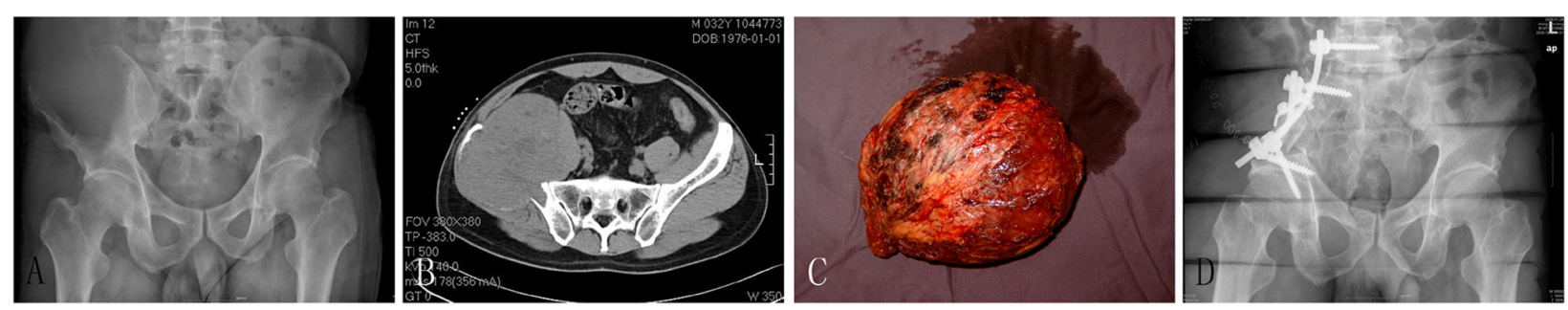

Fig. 2 Case presentation of a 32-year-old male presented a persistent pain of the left ilium for 3 months. a Osteolytic lesions can be observed in the left ilium on the plain film. $\mathbf{b}$ CT scan shows the tumor involvement in the ilium where a huge soft tissue mass formed. c The tumor was resected widely, and the inferior cut line was at the normal bone above the acetabulum. $\mathbf{d}$ Screws and rods were used for reconstruction of the pelvic ring after tumor resection 

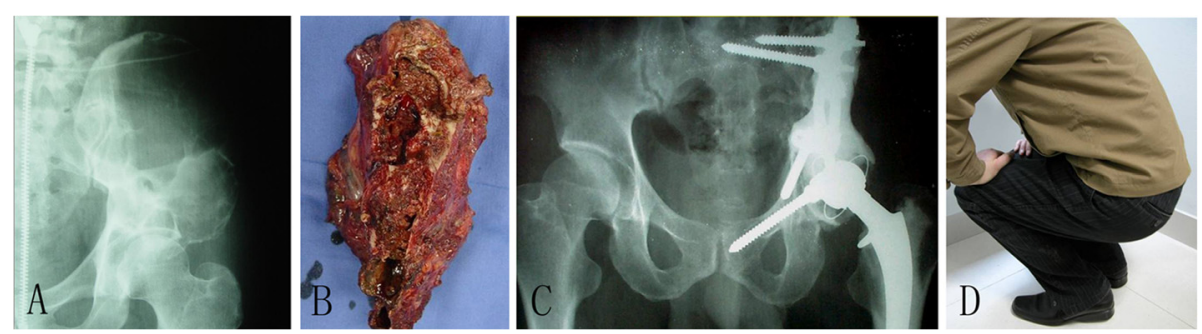

Fig. 3 Case presentation of a 34-year-old man who mainly complained about pain in the left hip area for 5 months which was aggravated over the previous 1 week. a Osteolytic lesions can be observed in the left ilium and periacetabular region on the plain film. $\mathbf{b}$ The tumor was resected widely, and the cut line was at the normal bone. c A plain film at 2 years after operation shows no recurrence. $\mathbf{d}$ The patient has good function without any discomfort in the last follow-up

Four patients (cases 2, 4, 20, and 26) with wound healing problems were managed by debridement with eventual healing between 4 and 6 weeks. Dislocation of the hip occurred in 1 patient (case 13) with femoral head exclusion after resection of pelvic tumors around the acetabulum, and she got local stability after long periods of bed rest. This patient could walk with crutches with no pain at the most recent follow-up. One patient (case 12) with a delayed infection eventually healed after wound debridement for three times and long-term antibiotic use.

GCT locations in the pelvis seem to be an influence factor regarding the surgical complications and MSTS functional score. Surgical complications were much more common, and the MSTS functional score was lower when the GCTs involved the acetabulum than those in the ilium or pubis/ischium $(p=0.010, p=0.005)$ (Table 2).

The mean MSTS functional score at the last follow-up was 22.8 (range 14-28) with a mean score of 25.4 for 8 patients who underwent intralesional surgery and 21.9 for 21 patients treated with wide resection. Some parameters were used for statistical analyses between group A (intralesional surgery) and group B (wide resection), but significant difference could only be found in the MSTS functional score $(p=0.024)$ (Table 3$)$.

\section{Discussion}

Giant cell tumors of the bone rarely affect the pelvis, with an incidence of only 1.5 to $6.1 \%$ of bone GCTs [4-6]. Only 73 pelvic lesions had reported in literatures from 1949 to 1999 [7], and the largest number of cases with pelvis GCTs presented in one study was no more than 30 . In this study, 29 patients with pelvis GCTs from five institutions had been collected for analysis. To our knowledge, it is the largest number of cases in the existing literatures as well as the only multi-center study on pelvis GCTs.

The local recurrence rate for giant cell tumors of the pelvis seems to be higher than that of any other location in the skeleton for western or eastern [23, 24], due to the complex anatomy and the large size these lesions can attain before diagnosis. The local recurrence rate can be as high as $43 \%$ for therapeutic options other than wide resection [7, 13-16, 25]. Sanjay et al. [16] reported that 3 of 15 patients with pelvic GCTs had local recurrence after intralesional surgery, whereas no one recurred in 2 patients who underwent wide resection. Balke et al. [13] reported that 1 of 16 patients with pelvic GCTs suffered local recurrence after intralesional surgery, whereas none of 3 patients with wide resection recurred. Guo et al. [8] reported that 4 of 13 patients with pelvic GCTs had local recurrence after intralesional surgery, whereas 0 of 14 patients suffered recurrence after wide resection. In our study, 2 of the 21 patients who underwent wide resection had local recurrences, whereas one of 8 patients who underwent intralesional surgery had local recurrence. No significant difference in recurrence was found between intralesional surgery and wide resection $(p=0.814)$. It is generally accepted that wide resection can reduce the risk of recurrence because the adjacent tissue can be resected with the tumor to achieve an adequate resection margin. For some pelvic GCTs, wide resection is hard to perform owing to the complex anatomic structures in the pelvis. Intralesional surgery is an adequate option for some patients based on careful preoperative planning. Initial surgical treatment is of great importance to patients on account of the fact that recurrence of the tumor may make it unresectable; as a consequence, it is vital for patients with pelvic GCTs to be operated by surgeons with sufficient knowledge of bone tumor. The tumor size and Campanacci classification should be taken into account in the preoperative plan. If the tumor is not limited to one region defined by the Enneking and Dunham classification system for pelvic tumors, we believe wide resection could be a reasonable choice. If the Campanacci classification is grade III that the bone walls are destroyed by the tumor and a large soft tissue mass forms, wide resection is recommended to ensure a safe margin.

Treatment options for pelvic GCTs include denosumab, serial embolization, interferon, radiation therapy, 
Table 2 The statistic analyses of 29 pelvic GCT patients basing on the location group

\begin{tabular}{|c|c|c|c|c|c|}
\hline Categories & Type $I^{a}$ & Type $\|^{a}$ & Type $1 \|^{a}$ & Total & $p$ \\
\hline Number, $n(\%)$ & $10(34.5)$ & $11(37.9)$ & $8(27.6)$ & $29(100)$ & - \\
\hline Age, year, means (SD) & $37.2(9.3)$ & $36.8(7.9)$ & $45.1(9.5)$ & $39.2(9.3)$ & 0.106 \\
\hline Age group, $n(\%)$ & & & & & 0.175 \\
\hline$<20$ & $0(0)$ & $1(9.1)$ & $0(0)$ & $1(3.4)$ & \\
\hline $20 \sim 29$ & $1(10.0)$ & $0(0)$ & $1(12.5)$ & $2(6.9)$ & \\
\hline $30 \sim 39$ & $6(60.0)$ & $5(45.5)$ & $1(12.5)$ & $12(41.4)$ & \\
\hline $40 \sim 49$ & $1(10.0)$ & $5(45.5)$ & $3(37.5)$ & $9(31.0)$ & \\
\hline$\geq 50$ & $2(20.0)$ & $0(0)$ & $3(37.5)$ & $5(17.2)$ & \\
\hline Sex, $n(\%)$ & & & & & 0.160 \\
\hline Male & $3(30.0)$ & $5(45.5)$ & $6(75.0)$ & $14(48.3)$ & \\
\hline Female & $7(70.0)$ & $6(54.5)$ & $2(25.0)$ & $15(51.7)$ & \\
\hline Treatment, $n(\%)$ & & & & & 0.691 \\
\hline$S(I L)$ & $2(20.0)$ & $4(36.4)$ & $2(25.0)$ & $8(34.5)$ & \\
\hline$S(W)$ & $8(80.0)$ & $7(63.6)$ & $6(75.0)$ & $21(31.0)$ & \\
\hline Follow-up, months, means (SD) & $62.2(38.0)$ & $75.5(59.9)$ & $38.3(15.3)$ & $59.0(42.7)$ & 0.215 \\
\hline Recurrence, $n(\%)$ & & & & & 0.065 \\
\hline Exist & $0(0)$ & $3(27.3)$ & $0(0)$ & $3(10.3)$ & \\
\hline None & $10(100)$ & $8(72.7)$ & $8(100)$ & $26(89.7)$ & \\
\hline Complication, $n(\%)$ & & & & & 0.010 \\
\hline Exist & $1(10.0)$ & $6(54.5)$ & $0(0)$ & $7(24.1)$ & \\
\hline None & $9(90.0)$ & $5(45.5)$ & $8(100)$ & $22(75.9)$ & \\
\hline MSTS, means (SD) & $23.6(2.4)$ & $20.2(4.3)$ & $25.5(2.3)$ & $22.8(3.8)$ & 0.005 \\
\hline
\end{tabular}

$S$ (IL) intralesional curettage, $S(W)$ wide resection

${ }^{a}$ According to the classification system of Enneking and Dunham

and surgical treatment. Denosumab is a fully humanized monoclonal antibody against the RANK ligand which has demonstrated significant activity in patients with unresectable or recurrent GCTs in the bone [9]. Denosumab was approved by the FDA for the treatment of adults and skeletally mature adolescents with GCT of the bone that is unresectable or where surgical resection is likely to result in severe morbidity in 2013. However, patients did not accept the denosumab treatment in China because the denosumab was not approved by CFDA and the patients' agreement was hard to get. Although serial embolization [10] and interferon [11] have been accepted in the GCT treatment, it is still necessary to perform surgical treatment thereafter. The obvious advantage of radiation therapy is the avoidance of additional surgical morbidity, while its disadvantages include local effects of radiation therapy and the potential for radiation-induced sarcoma. Leggon et al. [7] reviewed literatures of pelvic and sacral GCTs, which revealed that patients treated with radiation therapy had an incidence of $44 \%$ of local recurrence and $12 \%$ of radiation-induced sarcoma.

Therefore, it is quite clear that surgery plays an unparalleled role in the pelvis GCT treatments. It is widely accepted that intralesional surgery can spare nerve roots, pelvic support, and the hip and visceral structures, whereas it has a high risk of local recurrence, even for a recurrent GCT. Various adjuvant modalities have been used to supplement intralesional surgery in order for a low recurrence rate, which include the use of cytotoxic agents such as phenol [16], zinc chloride [26], ethanol [14], and physical adjuvants such as polymethylmethacrylate [13, 14, 27], cryosurgery [13], microwave inactivation [28], and a high-speed burr drill [13, 14]. Besides, a study shows that curettage based on CT classification can also lower the recurrence rate [29]. In this series, microwave inactivation had been employed for 3 patients before intralesional surgery and no recurrence occurred. It is obvious that microwave inactivation is performed before surgery other than other adjuvant treatments. The inactived tumor tissues do not have viable cells during curettage in theory. The difficulties for this method include protection of the surrounding normal tissues and complete inactivation for the whole tumor tissue. Furthermore, GCTs did not destroy the subchondral bone in the acetabular area for these 3 patients. We used polymethylmethacrylate for 2 patients in 
Table 3 The statistic analyses of 29 pelvic GCT patients basing on the treatment group

\begin{tabular}{lllll}
\hline Categories & $\mathrm{S}(\mathrm{IL})$ & $\mathrm{S}(\mathrm{W})$ & Total & $p$ \\
\hline Number, $n$ (\%) & $8(27.6)$ & $21(72.4)$ & $29(100)$ & - \\
Age, year, means (SD) & $34.8(9.0)$ & $41.0(9.0)$ & $39.2(9.3)$ & 0.732 \\
Age group, $n$ (\%) & & & & 0.190 \\
$\quad<20$ & $1(12.5)$ & $0(0)$ & $1(3.4)$ & \\
20 29 & $0(0)$ & $2(9.5)$ & $2(6.9)$ & \\
30 39 & $5(62.5)$ & $7(33.3)$ & $12(41.4)$ & \\
40 49 & $2(25.0)$ & $7(33.3)$ & $9(31.0)$ & \\
$\quad$ 250 & $0(0)$ & $5(23.8)$ & $5(17.2)$ & \\
Sex, $n$ (\%) & & & & 0.035 \\
$\quad$ Male & $1(7.1)$ & $13(92.9)$ & $14(48.3)$ & \\
Female & $7(46.7)$ & $8(53.3)$ & $15(51.7)$ & \\
Location, ${ }^{a} n$ (\%) & & & & 0.691 \\
I & $2(25.0)$ & $8(38.1)$ & $10(34.5)$ & \\
II & $4(50.0)$ & $7(33.3)$ & $11(52.4)$ & \\
III & $2(25.0)$ & $6(28.6)$ & $8(27.6)$ & \\
Follow-up, months, & $85.1(52.1)$ & $49.3(35.4)$ & $59.0(42.7)$ & 0.056 \\
means (SD) & & & & 0.814 \\
Recurrence, $n$ (\%) & & & & \\
Exist & $1(12.5)$ & $2(9.5)$ & $3(10.3)$ & \\
None & $7(87.5)$ & $19(90.5)$ & $26(89.7)$ & \\
Complication, $n$ (\%) & & & & 0.635 \\
$\quad$ Exist & $1(12.5)$ & $6(28.6)$ & $7(24.1)$ & \\
$\quad$ None & $7(87.5)$ & $15(71.4)$ & $22(75.9)$ & \\
MSTS, means (SD) & $25.4(3.6)$ & $21.9(3.5)$ & $22.8(3.8)$ & 0.024 \\
\hline
\end{tabular}

$S$ (IL) intralesional curettage, $S(W)$ wide resection

${ }^{a}$ According to the classification system of Enneking and Dunham

order for filling and inactivation, one of which suffered recurrence. The high-speed burr drill was employed in 2 patients to deal with residual GCT cells that may remain on the surface of the curettage cavity, and no recurrence occurred. We did not use nitrogen or other chemicals for the sake of avoiding chemical injury to the surrounding tissues from leaking adjuvants. Wide resection has been thought to minimize the chance of local recurrence, which is true for tumors in regions I and III, rather than the ones in region II. There is no doubt that wide resection is difficult to perform for large-size pelvic GCTs, especially those involving region II. An increased surgical morbidity is usual even for an experienced bone tumor surgeon.

The MSTS 93 score had been applied for patients' functional evaluation in this study. Compared with intralesional surgery, the function of the patients who accepted wide resection appeared worse. However, it was not absolute for all patients that most patients who accepted wide resection for GCTs in regions I and III had good functions. For the GCTs involving region II or more than one region, favorable functional results were hard to achieve although some specialized prostheses had been developed to reconstruct the defect after acetabular bone resection. In this series, we preferred to use screws, titanium rods, and bone cement to reconstruct the integrity of the pelvis based on our experience. The advantages of this method include simple instruments, flexible fixed way, and relatively stable mechanical strength. The disadvantages include fixation loosening and breakup. The fixation-related complications were not observed in our patients, and most patients who accepted pelvis reconstruction could resume their routine activities within 3 months after surgery.

As we know, complications are common for pelvis tumor because of wide wound exposure, extensive soft tissue stripping, implant existence, local hematoma formation, and poor skin flap blood supply [30]. In this study, wound healing disturbance was the major complication, which occurred in 4 patients who underwent wide resection and pelvis reconstruction. Limb shortening, delayed infection, and dislocation happened in 3 patients, respectively. It is obvious that patients with GCTs in region II had a higher incidence of complication compared with those in regions I and III. For reducing complications, it is necessary to protect the skin flap and reduce soft tissue tension. Otherwise, drainage is very important for postoperative care.

It is necessary to alert readers to be aware of the limitations of this study. Firstly, the number of patients is still small although it is the largest series among the reports owing to the fact that pelvic GCTs are rare. It is hard to make any definitive statements regarding the differences of recurrences, complications, and postoperative function among different treatments without large sample statistical analysis. Secondly, this is a multicentric retrospective study and the patients' treatments were decided by five experienced bone tumor surgeons, respectively; consequently, the differences among surgical technologies cannot be avoided. Nevertheless, consensuses of treatments had been made by these surgeons, and postoperative situations of different treatment methods may provide valuable information for surgeons in the decisionmaking process. Thirdly, the minimum follow-up is short and additional local recurrences might occur with longer follow-ups. Nevertheless, $70 \%$ of local recurrences occur within 2 years [31].

Treatment of pelvic GCTs remains a challenge for surgeons, especially for the ones involving region II. In this study, patients with GCTs that did not destroy the subchondral bone in region II achieved good functions and suffered no recurrence by undergoing microwave inactivation before intralesional surgery. Therefore, it could be an alternative treatment for some patients, although it should be determined on the basis of sufficient clinical 
evidence; as a consequence, a prospective study about the clinical efficacy of microwave inactivation for GCTs has already been launched by our team. It has great difficulties to achieve balance among recurrence, complications, and functions for the GCTs with benign histology and aggressive biological behavior; thus, detailed communication with patients is essential during the decisionmaking process.

\section{Conclusions}

There are only a few case reports in the literature and no large numbers of clinical trials about treatment of, and research into, pelvic GCTs. In this study, low recurrence rate and favorable postoperative functional results of pelvic GCTs were obtained successfully through intralesional curettage, combined with a meticulous preoperative planning. Due to the small sample size of this study, the results should be examined cautiously. Larger, high-quality clinical trials are required to strengthen and verify these conclusions.

\section{Consent}

The written informed consent was obtained from the patient for publication of this report and any accompanying images.

\section{Competing interests}

The authors declare that they have no competing interests.

\section{Authors' contributions}

$\mathrm{XY}$ and $\mathrm{YH}$ carried out the studies, participated in collecting the data, and helped draft the manuscript. KZ performed the data analysis, participated in its design, and drafted the manuscript. ZW, SW, and ZY conceived of the study and participated in its design and coordination. All authors read and approved the final manuscript.

\section{Acknowledgements}

The authors thank Jinghua Wang and Xianjia Ning (Department of Epidemiology, Tianjin Neurological Institute, Tianjin, China) for the statistical analyses.

\section{Author details \\ ${ }^{1}$ Second Military Medical University, Shanghai, China. ${ }^{2}$ Department of Orthopaedics, The General Hospital of Jinan Military Commanding Region, Jinan, Shandong, China. ${ }^{3}$ Department of Bone Oncology, Tianjin Hospital, Tianjin, China. ${ }^{4}$ Department of Orthopaedics, Xijing Hospital, Fourth Military Medical University, Xian, Shannxi, China. ${ }^{5}$ Department of Orthopaedics, Jinling Hospital, Clinical School of Medical College, Nanjing University, Nanjing, Jiangsu, China. ${ }^{6}$ Department of Orthopaedics, Second Affiliated Hospital, School of Medicine, Zhejiang University, Hangzhou, Zhejiang, China. ${ }^{7}$ Giant Cell Tumor Group of China (GTOC), Beijing, China.}

Received: 15 October 2015 Accepted: 29 March 2016

Published online: 05 April 2016

\section{References}

1. Karpik M. Giant cell tumor (tumor gigantocellularis, osteoclastoma) epidemiology, diagnosis, treatment. Ortop Traumatol Rehabil. 2010;12:207-15.

2. Trieb K, Bitzan P, Lang S, Dominkus M, Kotz R. Recurrence of curetted and bone-grafted giant-cell tumours with and without adjuvant phenol therapy. Eur J Surg Oncol. 2001;27:200-2.

3. Boons HW, Keijser LC, Schreuder HW, Pruszczynski M, Lemmens JA, Veth RP. Oncologic and functional results after treatment of giant cell tumors of bone. Arch Orthop Trauma Surg. 2002;122:17-23.
4. Reid R, Banerjee SS, Sciot R. Giant cell tumour. In: Fletcher CDM, Unni KK, editors. Pathology and genetics of tumours of soft tissue and bone. Lyon, France: IARC Press; 2002. p. 309-13.

5. Schajowicz F. Giant cell tumor. In: Schajowicz F, Sundaram M, editors. Tumors and tumorlike lesions of bone. New York: Springer-Verlag; 1996. p. 257-95.

6. Unni KK. Giant cell tumor. In: Unni KK, Dahlin DC, editors. Dahlin's bone tumors: general aspects and data on 11,087 cases. Philadelphia: LippincottRaven; 1996. p. 263-89.

7. Leggon RE, Zlotecki R, Reith J, Scarborough MT. Giant cell tumor of the pelvis and sacrum: 17 cases and analysis of the literature. Clin Orthop Relat Res. 2004;423:196-207.

8. Guo W, Sun X, Zang J, Huayi Q. Intralesional excision versus wide resection for giant cell tumor involving the acetabulum: which is better? Clin Orthop Relat Res. 2012;470:1213-20.

9. Chawla S, Henshaw R, Seeger L, Choy E, Blay JY, Ferrari S, Kroep J, Grimer R, Reichardt P, Rutkowski P, Schuetze S, Skubitz K, Staddon A, Thomas D, Qian $Y$, Jacobs I. Safety and efficacy of denosumab for adults and skeletally mature adolescents with giant cell tumour of bone: interim analysis of an open-label, parallel-group, phase 2 study. Lancet Oncol. 2013;14:901-8.

10. Onishi H, Kaya M, Wada T, Nagoya S, Sasaki M, Yamashita T. Giant cell tumor of the sacrum treated with selective arterial embolization. Int J Clin Oncol. 2010:15:416-9.

11. Wei F, Liu X, Liu Z, Jiang L, Dang G, Ma Q, Dang L. Interferon alfa-2b for recurrent and metastatic giant cell tumor of the spine: report of two cases. Spine (Phila Pa 1976). 2010;35:E1418-1422.

12. Caudell JJ, Ballo MT, Zagars GK, Lewis VO, Weber KL, Lin PP, Marco RA, ElNaggar AK, Benjamin RS, Yasko AW. Radiotherapy in the management of giant cell tumor of bone. Int J Radiat Oncol Biol Phys. 2003;57:158-65.

13. Balke M, Streitbuerger A, Budny T, Henrichs M, Gosheger G, Hardes J. Treatment and outcome of giant cell tumors of the pelvis. Acta Orthop. 2009;80:590-6.

14. Donati D, Wafa H, Di Bella C, Colangeli M, Colangeli S, Bertoni F. Management of pelvic giant cell tumours involving the acetabular bone. Acta Orthop Belg. 2008;74:773-8.

15. Osaka $\mathrm{S}$, Toriyama $\mathrm{S}$. Surgical treatment of giant cell tumors of the pelvis. Clin Orthop Relat Res. 1987;222:123-31.

16. Sanjay BK, Frassica FJ, Frassica DA, Unni KK, McLeod RA, Sim FH. Treatment of giant-cell tumor of the pelvis. J Bone Joint Surg Am. 1993;75:1466-75.

17. Enneking WF, Dunham WK. Resection and reconstruction for primary neoplasms involving the innominate bone. J Bone Joint Surg Am. 1978;60:731-46.

18. Gradinger R, Rechl H, Hipp E. Pelvic osteosarcoma: resection, reconstruction, local control, and survival statistics. Clin Orthop Relat Res. 1991;270:149-58.

19. Nilsonne U, Kreicbergs A, Olsson E, Stark A. Function after pelvic tumour resection involving the acetabular ring. Int Orthop. 1982;6:27-33.

20. Campanacci M. Giant cell tumor. In: Campanacci M, Enneking WF, editors. Bone and soft tissue tumors: clinical features, imaging, pathology and treatment. New York: Springer-Verlag; 1999. p. 99-136.

21. Tang X, Guo W, Yang R, Tang S, Ji T. Risk factors for blood loss during sacral tumor resection. Clin Orthop Relat Res. 2009;467:1599-604.

22. Enneking WF, Dunham WK, Gebhardt MC, Malawar M, Pritchard DJ. A system for the functional evaluation of reconstructive procedures after surgical treatment of tumors of the musculoskeletal system. Clin Orthop Relat Res. 1993;286:241-6.

23. Campanacci M, Baldini N, Boriani S, Sudanese A. Giant-cell tumor of bone. J Bone Joint Surg. 1987;69A:106-14

24. Sung HW, Kuo DP, Shu WP, Chai YB, Liu CC, Li SM. Giant-cell tumor of bone: analysis of two hundred and eight cases in Chinese patients. J Bone Joint Surg. 1982;64A:755-61.

25. Kattapuram AS, O'Donnell RJ, Huszar M, Rosenberg AE, Kattapuram SV, Mankin HJ. Surgical management of innominate giant cell tumor. Clin Orthop Relat Res. 1996;329:281-7.

26. Zhen W, Yaotian H, Songjian L, Ge L, Qingliang W. Giant-cell tumour of bone. The long-term results of treatment by curettage and bone graft. J Bone Joint Surg Br. 2004:86:212-6.

27. Yu X, Xu M, Xu S, Qing S. Clinical outcomes of giant cell tumor of bone treated with bone cement filling and internal fixation, and oral bisphosphonates. Oncology Letters. 2013;5:447-51.

28. Hu YC, Ji JT, Lun DX. Intraoperative microwave inactivation in-situ of malignant tumors in the scapula. Orthop Surg. 2011;3:229-35. 
29. Puthoor DK, Puthezhath K. Management of giant cell tumor of bone: computerized tomography based selection strategy and approaching the lesion through the site of cortical break. Orthop Surg. 2012;4:76-82.

30. Capanna R, van Horn JR, Guernelli N, Briccoli A, Ruggieri P, Biagini R, Bettelli G, Campanacci M. Complications of pelvic resections. Arch Orthop Trauma Surg. 1987:106:71-7.

31. Blake SM, Gie GA. Large pelvic giant cell tumor: a case report and a review of current treatment modalities. J Arthroplasty. 2004;19:1050-4.

Submit your next manuscript to BioMed Central and we will help you at every step:

- We accept pre-submission inquiries

- Our selector tool helps you to find the most relevant journal

- We provide round the clock customer support

- Convenient online submission

- Thorough peer review

- Inclusion in PubMed and all major indexing services

- Maximum visibility for your research

Submit your manuscript at www.biomedcentral.com/submit 